\title{
COLABORADORES
}

\section{Relação de pareceristas ad hoc (2020-2021)}

Educação e Pesquisa agradece aos especialistas abaixo relacionados, que colaboraram com a revista no período de out. 2020 a out. 2021.

Acacia Zeneida Kuenzer
Ademir Donizeti Caldeira
Adenize Costa Acioli
Adriana Bauer
Adriana Melo
Adriana Santiago Rosa Dantas
Adriane Knoblauch
Adriano Vieira de Carvalho
Agnaldo Arroio
Aguinaldo Cesar Surdi
Alan Augusto Moraes Ribeiro
Alberto Moreno-Doña
Alex Bellucco do Carmo
Alexander Armando Cordoves
Santiesteban
Alexandre Pianelli Godoy
Alexandre Ramos de Azevedo
Alexandre Saul
Alexandre Toaldo Bello
Alexsandro do Nascimento Santos
Alfredo Gabriel Buza
Alice Casimiro Lopes
Alice Ribeiro Casimiro Lopes
Alicia Beatriz Gutiérrez
Aline de Morais Limeira Pasche
Aline Silva Dejosi Nery
Allan Kenji Seki
Américo Rodrigues de Almeida Neto
Amurabi Oliveira
Ana Alice Vilas Boas
Ana Beatriz Gomes Pimenta de
Carvalho

$\begin{array}{ll}\text { Ana Claudia D. Capistrano de Oliveira } & \text { Benerval Pinheiro Santos } \\ \text { Ana Claudia Urban } & \text { Bianca Rigamonti Valeiro Garcia } \\ \text { Ana Cristina Coll Delgado } & \text { Bruna Soares Polachini } \\ \text { Ana Elisa Spaolonzi Queiroz Assis } & \text { Bruno Nunes Batista } \\ \text { Ana Gladcheff } & \text { Camila Ferreira da Silva } \\ \text { Ana Lúcia Calbaiser da Silva } & \text { Candido Alberto da Costa Gomes } \\ \text { Ana Lúcia Cunha Duarte } & \text { Carlos Betlinski } \\ \text { Ana Lúcia Sarmento Henrique } & \text { Carlos Herold Junior } \\ \text { Ana Maria Di Grado Hessel } & \text { Carola Gabriela Sepúlveda Vásquez } \\ \text { Ana Maria Fonseca de Almeida } & \text { Carolina Mostaro Neves da Silva } \\ \text { Ana Maria Nogales Vasconcelos } & \text { Carolina Rodrigues de Souza } \\ \text { Ana Paula Abrahamian de Souza } & \text { Caroline Fockink Ritt } \\ \text { Ana Paula dos Santos de Sá } & \text { Cássio José de Oliveira Silva Silva } \\ \text { Anderson de Carvalho Pereira } & \text { Catarina de Almeida Santos } \\ \text { André da Silva Mello } & \text { Catarina Mangas } \\ \text { André Dalben } & \text { Célia Cristina de Figueiredo Cassiano } \\ \text { André Luiz C. Gonçalves de Oliveira } & \text { Célia Regina Batista Serrão } \\ \text { Andre Luiz dos Santos } & \text { César Augusto Minto } \\ \text { André Rosalém-Signorelli } & \text { Cesar Lehmann Albornoz } \\ \text { Andréa Barbosa Gouveia } & \text { Charliton José dos Santos Macado } \\ \text { Andrea Cristiane Maraschin Bruscato } & \text { Christian Fernando Ribeiro } \\ \text { Andrea Cristina Versuti } & \text { Guimarães Vinci } \\ \text { Andréia Arruda Guidetti } & \text { Christiane Coutheux Trindade } \\ \text { Andréia Jaqueline Devalle Rech } & \text { Cibele Krause Lemke } \\ \text { Andrés Donoso Romo } & \text { Cibele Noronha de Carvalho } \\ \text { Andreza Barbosa } & \text { Cileda Santos Sant'Anna Perrella } \\ \text { Angela Maria Cristina Uchoa A. Branco } & \text { Clarice Monteiro Escott } \\ \text { Antonia Almeida Silva } & \text { Claudete Pagotto } \\ \text { Antonia Terra de Calazans Fernandes } & \text { Claudia Barcelos de Moura Abreu } \\ \text { Antonio Alberto Pereira } & \text { Cláudia Hilsdorf Rocha } \\ \text { Antonio Hilario Aguilera Urquiza } & \text { Cláudio Rodrigues da Silva } \\ \text { Barbara Lutaif Bianchini } & \text { Cleci T. Werner da Rosa } \\ & \end{array}$


Cleriston Izidro dos Anjos

Clériston Silva dos Anjos

Cristiane Machado

Cristiano Alberto Muniz

Cristiano Barbosa de Moura

Cristina Ferreira de Assis

Cristovam da Silva Alves

Cynthia Martínez-Garrido

Daielly Melina Nassif Mantovai

Daniel Campos de Carvalho

Daniel Ríos Muñoz

Daniel Tojeira Cara

Daniela Aparecida Eufrásio

Daniela da Costa Britto Pereira Lima

Daniele Cristina Souza

Danilo Martuccelli

David Antonio da Costa

Debora Cristina Goulart

Debora Cristina Piotto

Denilson Soares Cordeiro

Denise Madeira de Castro e Silva

Denize de Aguiar Xavier Sepulveda

Diana Patricia Ferreira de Santana

Diana Vidal

Diego dos Santos Reis

Dirce Aparecida Foletto Moraes

Dislane Zerbinatti Moraes

Eder Coelho Paula

Edilene Maria da Silva

Eduardo Di Deus

Elaine Prodócimo

Elenilton Vieira Godoy

Elias Manuel Said Hung

Élida Lopes Miranda

Elie George Guimarães Ghanem Jr.

Elio Carlos Ricardo

Elisabeth Mazeron Machado,

Elisangela Lazarou Tarraço

Elisangela Tarraco

Eliseo Alfonso Barca Lozano

Elma Júlia Gonçalves de Carvalho

Emilia Peixoto Vieira
Érica Aparecida Garrutti de Lourenço

Érica Santana Silveira Nery

Ermelinda Moutinho Pataca

Eromi Izabel Hummel

Etienne Barbosa Baldez

Evaldo Epiolli

Evelise Maria Labatut Portilho

Fabiana Cristina Frigieri de Vitta

Fabiana Silva Fernandes

Fabiany De Cássia Tavares Silva

Fábio Hoffmann Pereira

Faní Quitéria Nascimento Rehem

Fátima Niemeyer da Rocha

Fátima Suely Ribeiro Cunha

Fernanda de Jesus Costa

Fernanda Pinto de Aragão Quintino

Fernanda Rossi

Fernanda Sarturi

Fernando César Silva

Fernando Cezar Vieira Malange

Fernando Protetti

Fernando Rezende da Cunha Junior

Flávia dos Santos Soares

Flávia Inês Schilling

Flavia Pereira Xavier

Flávio Caetano da Silva

Flávio Santiago

Franciane Maria Araldi

Francisco García Marcos

Fredy Enrique González

Gardia Maria Vargas

George Coutinho

Geraldo Eustáquio Moreira

Géssica Priscila Ramos

Gilberto Ferreira da Silva

Giovanna Modé Magalhães

Gisele Belusso

Gladis Massini-Cagliari

Glauco da Silva Aguiar

Graciella Watanabe

Guilherme Gabriel Ballande Romanelli

Hartmut Günther
Helder Boska de Moraes Sarmento

Henrique César da Silva

Henrique Evaldo Janzen

Herman Fialho Fumiã

Hermengarda Alves Lüdke

Hivy Damasio Araújo Mello

Hormindo Pereira de Souza Junior

lara Tatiana Bonin

Ilka Miglio de Mesquita

Ingrid Hötte Ambrogi

Ione Ribeiro Valle

Iracema Santos do Nascimento

Íris Susana Pires Pereira

Isabel Melero Bello

Itale Luciane Cericato

Ivanildo Lubarino Piccoli dos Santos

Jaciara de Sá Carvalho

Jackson Gois da Silva

Jaqueline Dourado do Nascimento

Jefferson Mainardes

Jerusa Vilhena de Moraes

Joaquim ramos

Joedson Brito dos Santos

Joice Sousa Costa

Jonas Rangel de Almeida

Jorge Dorfman Knijnik

José Carlos Miguel

José Carlos Santos

José Cezinaldo Rocha Bessa

José Cláudio Sooma Silva

José Marcelino de Rezende Pinto

José Pedro Boufleuer

José Pedro Cerdeira

Júlia Catani

Juliana de Souza Silva

Julio César Tovar-Gálvez, Jurema Luzia de Freitas Sampaio, Jussara Cristina Barboza Tortella

Karina Carrasqueira Lopes

Karina Soledad Maldonado Molina

Karla Mendonça Menezes

Katia Adair Agostinho 
Katia Arillha Fiorentino Nanci

Katia Cristina Silva Forli Bautheney

Katia Cristina Stocco Smole

Kátia Liége Nunes Gonçalves

Katiuscia Cristina Vargas Antunes

Keila Deslandes

Keli Cristina Conti

Kimi Aparecida Tomizaki

Kristie Helene Novoa Durante

Leda Maria de Oliveira Rodrigues

Léia Teixeira Lacerda

Leonardo Borne

Leôncio José Gomes Soares

Leonilda do Nascimento da Silva

Lia da Rocha Lordelo

Lílian do Valle

Lisiane Machado de Oliveira-

Menegotto

Livia Suassuna

Lucas Nascimento Ferraz Costa

Lúcia Helena Sasseron

Lucia Maria Salgado dos Santos

Lombardi

Lúcia Pereira Leite

Luciana Esmeralda Ostetto

Luciana Garcia Mello

Luciana Paula Vieira de Castro

Lucila Maria Pesce de Oliveira

Luis Alberto Guzmán Palacios

Luís Paulo Leopoldo Mercado

Luiz Antonio Callegari Coppi

Luiz Eduardo P. Basto Tourinho Dantas

Luiz Roberto Alves

Maévi Anabel Nono

Manuel Alejandro Giovine

Marcelo Augusto Totti

Marcelo Rodrigues Conceição

Márcia Buss-Simão

Márcia Helena Alvim

Marcia Machado de Lima

Márcio de Oliveira

Marco Braga
Marcos Barbosa Oliveira

Marcos Fernando Ruiz Ruiz

Marcos Garcia Neira

Marcos Nascimento Magalhães

Marcus Vinícius Medeiros Pereira

Maria Amélia Rosário Santoro Franco

Maria Aparecida de Oliveira Freitas

Maria Aparecida Mello

Maria Beatriz Luce

Maria Cândida Borges de Moraes

Maria Cristina Cavaleiro

Maria da Conceição F. B. Sgadari Pas

Maria de Lourdes Bernartt

Maria Dilnéia Espíndola Fernandes

Maria do Carmo de Lacerda Peixoto

Maria Eugênia de L. Montes Castanho

Maria Eulina Pessoa de Carvalho

Maria Fernanda Rezende Nunes

Maria Graça Jacintho Setton

Maria Helena Câmara Bastos

Maria José da Silva Fernandes

Maria Jose Dias De Freitas

Maria Letícia B. Pedroso Nascimento

Maria Teresa Jacinto Sarmento

Maria Teresa Santos Cunha

María Victoria Mateo García

Maria Zenaide Alves

Marian Ávila de Lima e Dias

Marieta Gouvêa de Oliveira Penna

Marília Moschkovich

Marina Rezende Bazon

Marinez França de Souza

Mário Henrique Gomes

Mario Hernández Hernández-Nodarse

Mario Luiz Neves de Azevedo

Marisa Eugênia Melillo Meira

Maritania Salete Salvi Rafagnin

Marizete Lucini

Marta Genú Soares

Matheus Henrique da Fonsêca Barros

Matheus Monteiro Nascimento

Maurilane de Souza Biccas
Mauro Cavalcante Pequeno

Michele Viviene Carbinatto

Michelle Prazeres Cunha

Miguel Luiz Ambrizzi

Milton Rosa

Miriam Sonlleva Velasco

Mirian Jorge Warde

Mônica Appezzato Pinazza

Monica Villaça Gonçalves

Mylene Nogueira Teixeira

Nara Jorge Wellausen Vieira

Nathalie de Almenda Bressiani

Naylla Morais de Souxa

Neiva Furlin

Neli Maria Mengalli

Neusa Maria Dal Ri

Nicholas Davies

Nyuara Araújo da Silva Mesquita

Ocimar Munhoz Alavarse

Paloma Rezende de Oliveira

Patrício Carneiro Araújo

Patricio Lepe-Carrión

Paula Francisca da Silva

Paulo Vitor de Souza Pinto

Pedro Angelo Pagni

Pedro Ganzeli

Pedro Guilherme Rocha dos Reis

Pedro Jorge Caetano

Pedro Ribeiro Mucharreira

Poliana Bruno Zuin

Preciosa Fernandes

Priscila Monteiro Chaves

Raiane Florentino

Rainri Back

Raíssa de Oliveira Chappaz

Raquel Fontes Borghi

Raquel Lazzari Leite Barbosa

Raquel Lima

Raquel Souza

Regina Cândida Ellero Gualtieri

Reginaldo Celio Sobrinho

Reginaldo Fernando Carneiro 


Régis Henrique dos Reis Silva
Relma Urel Carbone Carneiro
Renata Marcílio Cândido
Renata Pontes Costa
Renato Abreu Lima
Renato Marcon Pugliese
Ricardo Boklis Golbspan
Ricardo Jorge De Sousa Cavalcanti
Rinaldo Molina
Rinaldo Voltolini
Rita de Cassia Gallego
Rita Santos Guimarães
Roberta Stangherlim
Roberto Rafael Dias da Silva
Robson Rocha de Souza Júnior
Rodison Roberto Santos
Rodrigo Diego de Souza
Rodrigo Lages e Silva
Rodrigo Saballa de Carvalho
Rogério Diniz Junqueira
Rogério Luis da Rocha Seixas
Rogério Vicente Ferreira
Romilda Costa Motta
Romilda Teodora Ens
Ronaldo Rosas Reis
Roni Cleber Dias de Menezes
Roraima Alves da Costa Filho
Rosa Aguilar Verastegui
Rosa Fátima de Souza Chaloba
Rosa Maria Ferreira da Silva
Rosana Amaro
Rosana Evangelista da Cruz
Rosana Louro Ferreira Silva
Ro

Rosângela Gavioli Prieto

Rosário Silvana Genta Lugli

Rosemeire Reis

Salomão Barros Ximenes

Sandra Maria Zákia Lian Sousa

Sandra Regina Simonis Richter

Sandra Unbehaum

Sergio Antonio Carlos

Sergio Baptista dos Santos

Sergio Paulino Abranches

Silvio Ricardo Gomes Carneiro

Silvio Ricardo Munari Machado

Simone Rocha Salomão

Simone Sarmento

Sonia Carbonell Alvares

Sonia Kramer

Sonia Maria Duarte Grego

Sonia Maria Rummert

Sonia Maria S.Corrêa de Souza Cruz

Sônia Teresinha Sousa Penin

Sônia Vanzella Castellar

Souzana Mizan

Stela Maria Fernandes Marques

Stephen M. Hart

Sueli Fanizzi

Sueli Lima Moreira

Suzana dos Santos Gomes

Suzana Lopes de Albuquerque

Sylvia De Chiaro

Tatiana Luiza Rech

Telma Ferraz Leal

Terezinha Oliveira

Terezinha Valim Oliver Gonçalves
Tícia Cassiany Ferro Cavalcante

Tulio Campos

Umberto de Andrade Pinto

Valéria Amorim Arantes

Valmir Flôres Pinto

Vanessa Dias Moretti

Vanessa Elias de Oliveira

Vanessa Teixeira Pipinis

Vania de Vasconcelos Gico

Velda Torres

Vera Lúcia Guerra

Vera Regina Oliveira Diehl

Vera Teresa Valdemarin

Verilda Speridião kluth

Vicente Zatti

Vinicio de Macedo Santos

Vinicius Catão de Assis Souza

Violante F. Magalhães

Vivian Batista da Silva

Vivian Martins

Viviane Fernandes F. Pinto

Viviane Potenza Guimarães Pinheiro

Viviane Toraci Alonso de Andrade

Wagner Rodrigues Silva

Wagner Xavier Camargo

Waldemar Ferreira Netto

Wania Regina Coutinho Gonzalez

Wilma Favorito

Wivian Weller

Yamila Irupé Nuñez

Zaqueu Vieira Oliveira

Zilda Gláucia Elias Franco

Zuleide Simas Silveira 